Published in final edited form as:

Respir Care. 2013 March ; 58(3): e31-e33. doi:10.4187/respcare.01897.

\title{
Pseudohypoxemia in a patient with chronic lymphocytic leukemia
}

\author{
Samuel Horr, M.D. ${ }^{1}$, Russell Roberson, M.D. ${ }^{2}$, and John W. Hollingsworth, M.D. ${ }^{1,3}$ \\ ${ }^{1}$ Department of Medicine, Duke University Medical Center \\ ${ }^{2}$ Department of Anesthesiology, Duke University Medical Center \\ ${ }^{3}$ Department of Immunology, Duke University Medical Center
}

\section{Keywords}

hypoxia; leukocyte larceny; red blood cell; white blood cell; platelet; pseudohypoxemia

\section{INTRODUCTION}

Arterial blood gas analysis is one of the most common tests performed on patients in intensive care units to measure arterial oxygen tension $\left(\mathrm{PaO}_{2}\right)$ as a means to determine adequate gas exchange. However, the partial pressure of oxygen in an arterial blood sample can be impacted by metabolically active cells including either white blood cells or platelets. We present a patient who had severe defects in measured arterial oxygen tension related to chronic lymphocytic leukemia.

\section{Case Summary}

A 75 year old man was transferred to our hospital for hypoxic respiratory failure requiring intubation after presenting with a 2 day history of progressive dyspnea and orthopnea. His past medical history was significant for chronic lymphocytic leukemia (CLL), prostate cancer, hypertension, and an abdominal aortic aneurism repair. The patient was initially admitted to an outside hospital with an acute myocardial infarction supported by a positive troponin I, reduced left ventricular ejection fraction on echocardiogram, and anuric renal failure. He required intubation and mechanical ventilation for severe hypoxemia and was transferred to our hospital.

He had no known drug allergies. His home medications include; metoprolol, furosemide, lisinopril, and aspirin. He had no history of tobacco, ethanol, or illicit drug use. On arrival to our intensive care unit the patient had a blood pressure of $105 / 55 \mathrm{mmHg}$, heart rate of 102 beats per minute, temperature of $36.6^{\circ} \mathrm{C}$ and oxygen saturation of $100 \%$ via pulse oximetry on $\mathrm{FiO}_{2}$ of $60 \%$. The patient was intubated and sedated. His exam was notable for clear breath sounds, normal heart sounds, and no jugular venous distention. No pitting edema or clubbing was identified. He had minimal urine output.

His laboratory data revealed an elevated pro-brain natriuretic peptide of $46,525 \mathrm{pg} / \mathrm{mL}$ (<850), CK MB of $6 \mathrm{ng} / \mathrm{mL}(<10)$, and an elevated troponin $\mathrm{T}$ of $0.22 \mathrm{ng} / \mathrm{mL}(<0.1)$. ECG

Corresponding Author: John W. Hollingsworth M.D., Division of Pulmonary, Allergy, and Critical Care Medicine, Department of Medicine, Duke University School of Medicine, DUMC 103004, 106 Research Drive, Durham, NC 27710, USA, Phone: (919)684-4588, Fax: (919) 684-5266; john.hollingsworth@duke.edu.

Conflict of Interest Statements: The authors have no conflict of interest to report related to the content of this case report. 
revealed sinus tachycardia, low voltage, and non-specific $\mathrm{T}$ wave changes. The complete blood count demonstrated a hemoglobin of $7.0 \mathrm{~g} / \mathrm{dL}$, platelet count of $73,000 / \mathrm{mm}^{3}$, and white blood cell count of $572,000 / \mathrm{mm}^{3}$ consistent with the patients diagnosis of CLL. A basic metabolic profile was significant for an elevated BUN of $51 \mathrm{mg} / \mathrm{dL}$ and creatinine of $2.2 \mathrm{mg} / \mathrm{dL}$. LDH was elevated to 769U/L (100-200) and uric acid and phosphorus levels were normal. An arterial blood gas obtained on $\mathrm{FiO}_{2}$ of $60 \%$ showed a $\mathrm{pH}$ of $7.28, \mathrm{PaCO}_{2}$ of $51 \mathrm{mmHg}$ and a $\mathrm{PaO}_{2}$ of $41 \mathrm{mmHg}$ that increased to $\mathrm{PaO} 2$ of $68 \mathrm{mmHg}$ on a $\mathrm{FiO}_{2}$ of $100 \%$. A chest radiograph showed mild pulmonary edema. An echocardiogram showed new global left ventricular dysfunction with an ejection fraction of $25 \%$, normal right ventricle, and mild mitral regurgitation. Mixed venous oxygen saturation $\left(\mathrm{SvO}_{2}\right)$ was $59 \%$.

Cardiogenic shock with secondary acute renal failure was the initial diagnosis given of his history of progressive dyspnea and orthopnea, recent elevated troponin, new severely reduced left ventricular dysfunction, and evidence of poor tissue perfusion with low $\mathrm{SvO}_{2}$ and poor urine output. The patient did not initially respond to intravenous diuretics and required dialysis for volume removal with resolution of his pulmonary edema. His hemodynamics improved and urine output normalized ; however, the patient remained profoundly hypoxemic as assessed by arterial blood gas analysis on mechanical ventilation. The patient continued to require $\mathrm{FiO}_{2}$ of $60 \%$ with oxygen saturations of $100 \%$ as measured with pulse oximetry with a discordant $\mathrm{PaO}_{2}$ of $48-72 \mathrm{mmHg}$ on arterial blood gas analysis. Because of a concern of leukostasis contributing to possible ventilation/perfusion mismatch and hypoxemia, leukopheresis was initiated.

Suspicions were raised for pseudohypoxemia because the pulse oximeter readings did not correlate with $\mathrm{PaO}_{2}$ values obtained on the arterial blood gas sampling. To specifically test for hypermetabolic activity and increased oxygen consumption related to leukocytosis, we first ran simultaneous arterial samples when our patient was on $100 \% \mathrm{FiO}_{2}$ at room temperature and on ice to slow metabolic activity of the leukocytes (Figure 1A). We observed dramatically improved $\mathrm{PaO}_{2}$ from samples preserved on ice. Next, we obtained an arterial sample on $100 \% \mathrm{FiO}_{2}$ and analyzed $\mathrm{PaO}_{2}$ over time when compared to that of a control patient (Figure 1B). We observed rapid rate of decline in $\mathrm{PaO}_{2}$ in our patient with leukocytosis, when compared to a control arterial sample.

After confirming our suspicion of pseudohypoxemia, this patient was weaned to $30 \% \mathrm{FiO}_{2}$ and remained with oxygen saturations of $98-100 \%$ via pulse oximetry without evidence of low tissue perfusion. He was liberated from the ventilator successfully the same day. He was subsequently begun on systemic chemotherapy for his CLL.

\section{DISCUSSION}

Pseudohypoxemia secondary to leukocytosis was first described in 1979 and was coined "leukocyte larceny" (1-2). This phenomenon is believed to be secondary to a high number of metabolically active white blood cells with an elevated consumption of the dissolved oxygen in arterial blood samples (3-4). It is recognized that both leukocytes and platelets account for the majority of the oxygen consumption in whole blood once removed from the body. The rate of oxygen consumption by white blood cells is typically clinically insignificant in the majority of patients with normal blood counts (5). Spuriously low $\mathrm{PaO}_{2}$ measurements are more common when either the white blood cell counts exceeds $50,000 / \mathrm{mm}^{3}$ or with severe thrombocytosis.

Patients with leukemia and hyperleukocytosis are at high risk for many causes of severe hypoxemia including: infection, pulmonary embolism, pulmonary leukostasis, leukemic infiltration, opportunistic neoplasms, hemorrhage, and drug-related toxicities (6); therefore, 
a thorough workup is required to rule out alternate causes of hypoxemia. Inconsistencies between measurements of hemoglobin oxygen saturation, as measured by pulse oximetry, and measurements of oxygen tension on arterial blood gas help to rule out these causes of hypoxemia (Figure 2). In this scenario, only a few possible diagnoses remain including methemoglobinemiaand pseudohypoxemia. To differentiate these causes compare the $\mathrm{PaO}_{2}$ to the $\mathrm{SpO}_{2}$. In methehemoglobinemia the $\mathrm{PaO}_{2}$ will be normal and the $\mathrm{SpO}_{2}$ will be falsely low. Pseudohypoxemia will present with normal $\mathrm{SpO}_{2}$ and falsely low $\mathrm{PaO}_{2}$. In contrast, carbon monoxide poisoning will present with a normal $\mathrm{PaO}_{2}$ and $\mathrm{SpO}_{2}$. Discordant values between $\mathrm{PaO}_{2}$ and $\mathrm{SpO}_{2}$ should be thoroughly investigated.

More accurate measurements of oxygen tension can be achieved by minimizing delay in processing arterial samples and preserving samples on ice. Previous work demonstrates that delayed processing of samples results in reduction of $\mathrm{PaO}_{2}$ in subjects with severe leukocytosis. Rapid cooling of arterial samples on ice will reduce the metabolic activity of the white blood cells resulting in a decreased rate of decline in oxygen concentrations (1-2) This method remains limited as considerable oxygen consumption occurs during sample cooling and will continue at a higher rate than normal despite the reduced metabolic activity (7). Other proposed methods to more accurately measure the $\mathrm{PaO}_{2}$ include either continuous arterial sampling or plasma sampling (8-9). Potassium cyanide can be used to block white blood cell metabolic activity, which will inhibit leukocyte larceny of dissolved oxygen. Finally, the addition of sodium fluoride to the sample may prevent leukocytes uptake of glucose and oxygen (2). These methods are technically challenging and are not widely available in a clinical setting. Pulse oximetry is thought to be the most reliable and clinically useful method to assess true oxygenation and remains an invaluable tool to assist in the diagnosis of pseudohypoxemia.

Our patient demonstrates that very high white blood cell counts falsely lower arterial blood gas measurements of oxygen tension. Astute clinicians should consider the diagnosis of pseudohypoxemia in the patient with white blood cell counts $>50,000 / \mathrm{mm}^{3}$ or severe thrombocytosis, high oxygen saturations on pulse oximetry, very low $\mathrm{PaO}_{2}$ on arterial blood gas and no alternative etiology of hypoxemia. Cooling (on ice) and rapid analysis of arterial samples may improve reliability of analysis of $\mathrm{PaO}_{2}$. As observed in our patient, pulse oximetry in the context of clinical evidence supporting adequate tissue perfusion may prove the most reliable method to assess oxygenation in patients with leukocyte larceny. Early diagnosis of pseuodohypoxemia could prevent unnecessary testing and prolonged mechanical ventilation.

\section{Acknowledgments}

The authors appreciate continued support from the NIH (ES016126, ES020426, and AI081672 to JWH).

\section{REFERENCES}

1. Hess CE, Nichols AB, Hunt WB, Suratt PM. Pseudohypoxemia secondary to leukemia and thrombocytosis. N Engl J Med. 1979; 301(7):361-363. [PubMed: 460325]

2. Fox MJ, Brody JS, Weintraub LR. Leukocyte larceny: A cause of spurious hypoxemia. Am J Med. 1979; 67(5):742-746. [PubMed: 292309]

3. Lele A, Mirski M, Stevens R. Spurious hypoxemia. Crit Care Med. 2005; 33(8):1854-1856. [PubMed: 16096465]

4. Sacchetti A, Grynn J, Pope A, Vasso S. Leukocyte larceny: spurious hypoxemia confirmed with pulse oximetry. J Emerg Med. 1990; 8(5):567-569. [PubMed: 2254603]

5. Cline MJ. Metabolism of a circulating leukocyte. Physiol Rev. 1965; 45(4):674-720. [PubMed: 5318995] 
6. Hildebrand FL Jr, Rosenow EC 3rd, Habermann TM, Tazelaar HD. Pulmonary complications of leukemia. Chest. 1990; 98(5):1233-1239. [PubMed: 2225971]

7. Shohat M, Schonfeld T, Zaizoz R, Cohen IJ, Nitzan M. Determination of blood gases in children with extreme leukocytosis. Crit Care Med. 1988; 16(8):787-8. [PubMed: 3396373]

8. Mizock BA, Franklin C, Lindesmith P, Shah PC. Confirmation of spurious hypoxemia using continuous blood gas analysis in a patient with chromic myelogenous leukemia. Leuk Res. 1995; 19(12):1001-1004. [PubMed: 8632655]

9. Charan NB, Marks M, Carvalho P. Use of plasma for arterial blood gas analysis in leukemia. Chest. 1994; 105(3):954-955. [PubMed: 8131577] 


\section{Teaching Points}

1. Pseudohypoxemia is a phenomenon observed in patients with significantly elevated white blood cell and/or platelet counts.

2. Clinicians should consider pseudohypoxemia in their differential diagnosis of patients with high oxygen saturations on pulse oximetry and very low $\mathrm{PaO}_{2}$ in patients with white blood cell counts $>50,000 / \mathrm{mm}^{3}$ or severe thrombocytosis and no alternative etiology of hypoxemia.

3. Cooling arterial samples and rapid analysis may improve reliability of analysis of $\mathrm{PaO}_{2}$ in patients with very high white blood cell counts.

4. Pulse oximetry is the most reliable method to clinically assess oxygenation in patients with leukocyte larceny. 

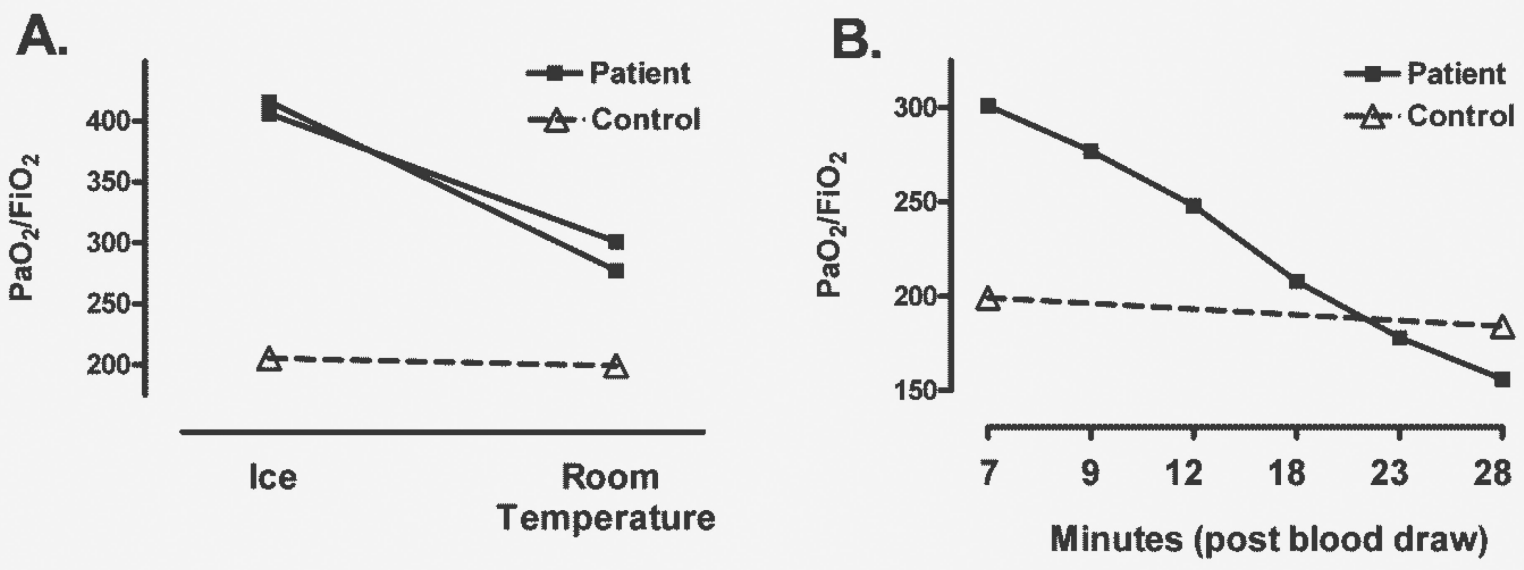

Figure 1. Arterial blood gas measurement of oxygen tension

Arterial samples were drawn from either our patient or a control subject on $100 \% \mathrm{FiO}_{2}$ and we report oxygen tension. (A) Samples were measured when either immediately placed on ice or at room temperature and oxygen tension was measured after five minutes (two repeats of our patient). (B) Arterial samples were then drawn and maintained at room temperature with serial measurements of oxygen tension of the same sample at 2-5 minute intervals for a total of 28 minutes after the time of blood draw. 


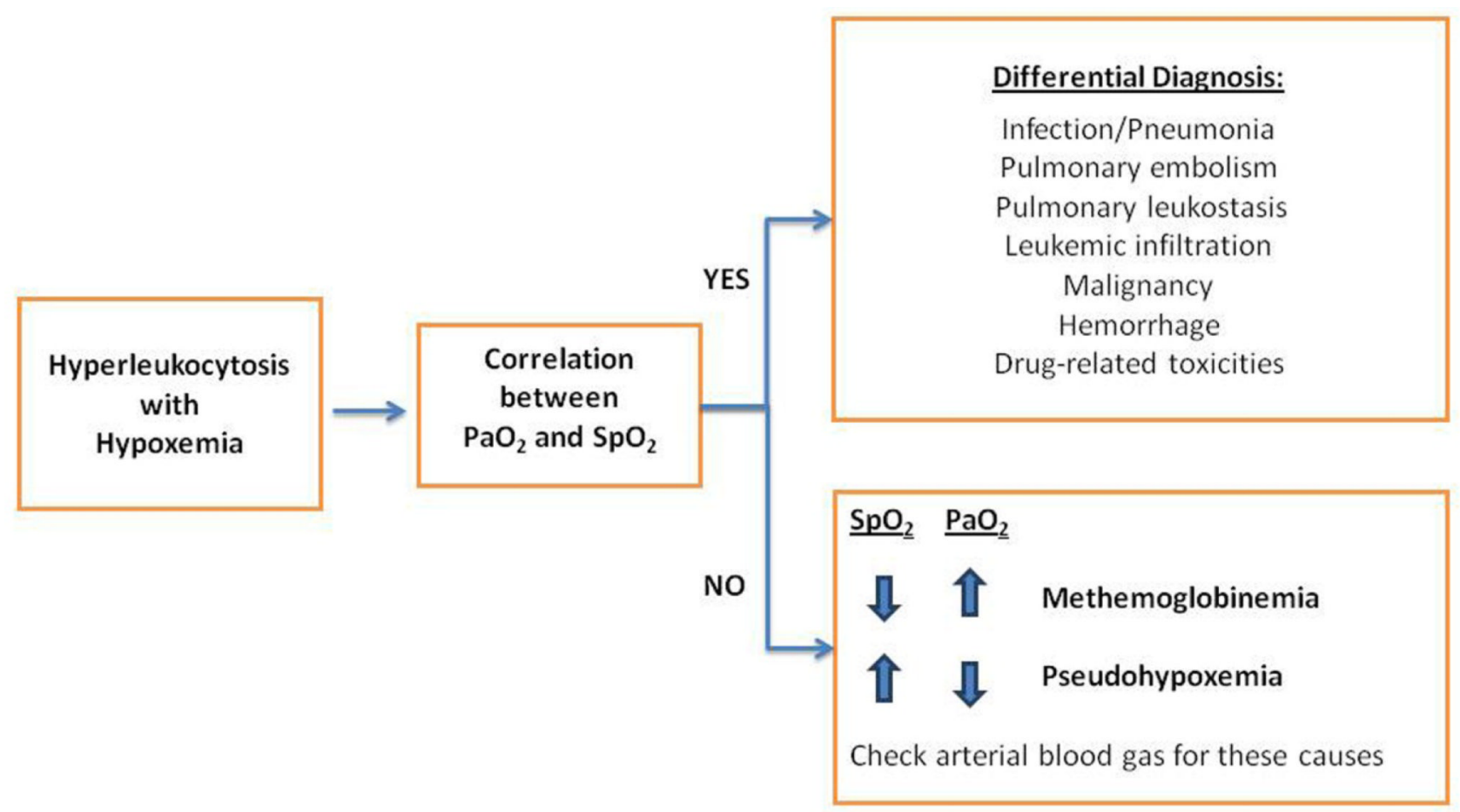

Figure 2. Diagnostic approach for patients with either hyperleukocytosis or thrombocytosis with hypoxemia

Discordant measurements of $\mathrm{PaO}_{2}$ and $\mathrm{SpO}_{2}$ should be thoroughly investigated. 